\title{
Human telomerase reverse transcriptase protein expression predicts tumor aggressiveness and survival in patients with clear cell renal cell carcinoma
}

Leili Saeednejad Zanjani 1, Zahra Madjd, 1,2*, Maryam Abolhasani 1,3, Arezoo Rasti 1, Ahmad Shariftabrizi ${ }^{4}$, Mojgan Asgari ${ }^{* 1,3}{ }^{3}$ Fint om du sender fulltekst på den i listen så vi får godkjent den

1. Oncopathology Research Centre, Iran University of Medical Sciences (IUMS), Tehran, Iran.

2. Department of Molecular Medicine, Faculty of Advanced Technologies in Medicine, Iran University of Medical Sciences, Tehran, Iran.

3. Hasheminejad Kidney Center, Iran University of Medical Sciences, (IUMS), Tehran, Iran.

4. Department of Nuclear Medicine and Molecular Imaging, State University of New York at Buffalo, Buffalo, NY 14223, USA.

\section{*Corresponding authors:}

Zahra Madjd (MD, PhD)

Oncopathology Research Center, Iran University of Medical Sciences (IUMS), Hemmat Street (Highway), Next to Milad Tower, Tehran14496-14530, Iran.

Phone: +982186703212

Fax: +982188622608

Email: Zahra.madjd@yahoo.com

Mojgan Asgari (MD)

Email: Mojgan_asgari@yahoo.com

Acknowledgments: This research was supported by a grant from Iran University of Medical Sciences (Grant \#25153).

Conflict of interest: The authors declare no conflict of interest.

Ethical approval: The study was approved by the Iran University of Medical Sciences. All procedures performed in this study were in accordance with the 1964 Helsinki Declaration and its later amendments. 
Informed consent: It was obtained at the time of sample collection in routine consent forms.

\begin{abstract}
Human telomerase reverse transcriptase (hTERT) is an active component of telomerase and responsible for its catalytic activity, associated with cell proliferation and differentiation. The present study, for the first time was conducted to evaluate the expression and prognostic significance of hTERT in different histological subtypes of renal cell carcinoma (RCC). Expression of hTERT was examined in 176 well-defined renal tumor samples including 113 (64.2\%) clear cell RCCs (ccRCCs), 32 (18.2\%) papillary RCCs, and 31 (17.6\%) chromophobe RCCs using immunohistochemistry on tissue microarrays. The association between hTERT expression and clinicopathologic parameters as well as survival outcomes were then analyzed. There was a statistically significant difference in terms of hTERT expression among various RCC subtypes. In ccRCC, increased expression of hTERT was significantly associated with advanced stage, higher grade, presence of microvascular invasion (MVI), lymph node invasion (LNI), and metastasis. Moreover, in the multivariate analysis, tumor stage and tumor size were independent predictors of the overall survival (OS). Additionally, expression of hTERT was found to be a significant predictor of worse OS $(\mathrm{P}=0.013)$ in the univariate analysis in ccRCC cases. In papillary carcinoma samples, significant association was detected between hTERT expression and tumor stage as well as metastasis, however, in chromophobe RCC, no significant association was found. We showed that hTERT protein expression was associated with more aggressive tumor behavior and more advanced disease in ccRCC patients. Also, hTERT may be a novel negative (?) prognostic indicator of the overall survival in these cases.
\end{abstract}

Key words: hTERT. Protein expression. Renal cell carcinoma (RCC). clear cell renal cell carcinoma (ccRCC). Tissue microarray (TMA). 


\section{Introduction}

Renal cell carcinoma (RCC) is the most common neoplasm of the adult kidney (80\% to 90\%), accounting for a total of $2 \%$ to $3 \%$ of adult malignancies, and is the most fatal urologic cancer [1]. The 2004 World Health Organization (WHO) classified RCC into several subtypes: the most common subtype is clear cell renal cell carcinoma (ccRCC), representing $75 \%$ to $80 \%$ of primary renal malignancies followed by papillary and chromophobe RCC accounting for $10 \%$ to $15 \%$ and $5 \%$ of renal cancers, respectively [2]. It is estimated that there will be 63,990 new cases of RCC in the United States and 14,400 related deaths in 2017[3]. Despite the recent advances in the diagnosis and treatment of RCC such as using tyrosine kinase and mTOR kinase inhibitors [4,5], up to $30 \%$ of RCC patients present with distant metastatic disease and $20 \%$ to $40 \%$ develop recurrent disease after surgery, therefore, RCC is still one of the deadliest forms of malignancies with a continued increase in incidence [6].

Human telomerase is a ribonucleoprotein enzyme consisting of two main components: catalytic component, human telomerase reverse-transcriptase (hTERT) and human telomerase RNA molecule (hTR), in addition to telomerase-associated proteins hTEP1, p23, Hsp90, and dyskerin. Telomerase adds the telomere repeat sequence (TTAGGG)n to the $3^{\prime}$ end of telomeres using its RNA as a template and hTERT [7]. Evidence shows that telomerase is expressed in $85 \%$ of malignancies, and the level of its activity is higher in advanced and metastatic tumors. While it is absent or barely detectable in most human normal somatic cells and in germ cells, stem cells, and cardiovascular cells express significant detectable levels of telomerase [8-10].

A previous study has shown that hTERT, an active component of telomerase and responsible for its catalytic activity, has other functions unrelated to its reverse transcriptase activity, such as increasing the anti-apoptotic capacity, enhancing DNA repair, maintaining stem cells and regulating gene expression [11].

A number of clinical studies have been performed to evaluate the association between telomerase activity and clinicopathological parameters in renal cancer showing that telomerase activity level correlates with progression of RCC [12-14]. However, we could not find any data regarding hTERT protein expression patterns and its association with clinicopathological parameters and survival in RCC patients.

In view of these facts, the present study was designed to investigate for the first time the expression levels and potential prognostic role of hTERT protein in a series of RCC clinical tumor samples, composed of clear cell, papillary, and chromophobe RCC using tissue microarray (TMA) method.

\section{Materials and methods}

\section{Patient characteristics and tumor samples}

A total of 176 paraffin-embedded tissues from RCC clinical tumor samples were included in this study. These specimens were collected from the Hasheminejad hospital, a major referral university-based urology-nephrology center in Tehran, Iran, in the time period of 2010 to 2015. 
All samples were collected from patients who had undergone radical nephrectomy and had no history of radiation therapy. These samples comprised of various subtypes of RCC including clear cell, papillary, and chromophobe RCC. The hematoxylin and eosin (H \& E) stained slides and medical archival records were retrieved to obtain clinicopathological parameters including age, gender, tumor size (maximum tumor diameter), tumor stage, and nuclear grade. In addition, the presence of necrosis, distant metastasis, the involvement of regional lymph nodes, renal vein, sinus and pelvis, Gerota's fascia, and microvascular invasion (MVI) were recorded. Information about patients' outcomes including the time between radical nephrectomy and cancer-related death or last follow-up (if death did not occur) were also recorded. The stage was defined based on the pTNM classification for renal cell carcinomas [15].

\section{Immunohistochemistry (IHC) for hTERT protein expression}

Representative tissue cores from formalin-fixed and paraffin-embedded RCC tumors were selected by an experienced pathologist and collected in a tissue microarray (TMA), as recently published [16].

Briefly, all TMA sections were deparaffinized at $60{ }^{\circ} \mathrm{C}$ for 20 minutes and dehydrated with graded alcohol. Endogenous peroxides and non-reactive staining were blocked with $3 \% \mathrm{H} 2 \mathrm{O} 2$ for 20 minutes at room temperature. After washing the tissue sections three times, antigen retrieval was performed by immersing the tissues in citrate buffer $(\mathrm{pH}=6.0)$ for 10 minutes in an autoclave. The tissue sections were incubated with primary antibody, anti-Telomerase reverse transcriptase antibody (ab183105, abcam, dilution: 1/500), overnight at $4^{\circ} \mathrm{C}$. TMA slides were then incubated with anti-rabbit/anti-mouse Envision (Dako, Denmark) as a secondary antibody for 30 minutes. Staining patterns were visualized by exposure to 3, 3'-diaminobenzidine (DAB, Dako) followed by counterstaining with hematoxylin visualize antigen (Dako). Finally, the slides were dehydrated in alcohol, cleared in xylene (Dako), and mounted for examination. In each run of the experiment, human tonsillar tissue was used as a positive control, and for a negative control, the primary antibody was replaced with Tris-buffered saline.

\section{Evaluation of immunostaining}

Immunostaining of telomerase reverse transcriptase (hTERT) was independently evaluated by two pathologists (M.A. and M.A.) who were blinded to the patients' outcome and pathological information. A consensus was achieved for all samples. The intensity of staining was scored by applying a semi-quantitative system, ranging from negative to strong as follows: $0=$ negative, $1=$ weak, 2 = moderate, and $3=$ strong. The percentage of positive cells was categorized according to the positive tumor cells as follows: Group 1: less than 25\% positive cells, Group 2: $25 \%$ to $50 \%$ positive cells, Group 3: $51 \%$ to $75 \%$ positive cells, and Group 4: more than $75 \%$ positive cells. To compare all the available data, we assigned an overall histochemical score (H-score) to each case by multiplying the intensity score by the percentage of positive cells, which yielded a range from 0 to 300. In this study, median $\mathrm{H}$ - score was chosen to categorize samples as with high or low telomerase reverse transcriptase expression.

\section{Statistical analysis}


Data were analyzed using the "statistical software SPSS, version 20.0. Armonk, NY: IBM Corp".We reported the categorical data as $\mathrm{N}(\%)$ and quantitative data as mean (SD). Comparison of hTERT expression in ccRCC, papillary, and chromophobe RCC samples was done using Kruskal-Wallis \& Mann-Whitney $U$ tests, for pairwise comparison between groups based on Bonferroni adjustment. Moreover, Pearson's $\chi 2$ and Spearman's correlation tests were used to analyze the significance of association and correlation between hTERT expression and clinicopathological parameters. Overall survival (OS) curves were drawn using the Kaplan-Meier method and log-rank test was used to compare the estimated curves between groups. The Cox proportional hazards regression model was applied to determine which variables affected OS. Variables that significantly affected survival in univariate analysis were included in multivariable analyzes. A $p$-value of $\leq 0.05$ was considered statistically significant.

\section{Results}

\section{Patient characteristics}

Of the total 176 samples included, $113(64.2 \%)$ were ccRCC, $32(18.2 \%)$ papillary, and $31(17.6 \%)$ chromophobe RCC. The study population consisted of $123(69.9 \%)$ male and $53(30.1 \%)$ female patients, with a male/female ratio of 2.3. The mean age of patients was $55(\mathrm{SD}=13)$ years (ranging from 25 to 82); 85 (48.3\%) patients were younger than 55 and 91 (51.7\%) over 55 years old.

Tumor size ranged from 1 to $21 \mathrm{~cm}$ in the largest diameter and tumors were classified into four groups: Group 1: 0 to $4 \mathrm{~cm} 34$ (19.3\%), Group 2: 4.1 to $7 \mathrm{~cm} 63$ (35.8\%), Group 3: 7.1 to $10 \mathrm{~cm}$ $40(22.7 \%)$, and Group 4: >10.1 $\mathrm{cm} 39(22.2 \%)$.

In this study, $90(51.1 \%)$ patients had low nuclear grade tumors, (grade II), $48(27.3 \%)$ grade III tumors, and 7 (4.0\%) high nuclear grade tumors (grade IV).

Moreover, 45 (25.6\%) cases were stage I, 13 (7.4\%) stage II, 103 (58.5\%) stage III, and 15 (8.5\%) stage IV.

Regional lymph node involvement was found in 12 (6.8\%) cases; 33 (18.8\%) cases had MVI, 100 $(56.8 \%)$ renal sinus fat involvement, and 74 (42.0\%) cases had tumor necrosis. Moreover, 37 $(21.0 \%)$ cases showed subsequent metastasis during the follow-up period. Other less frequent sites of involvement were as follow: renal vein invasion $(9 ; 5.1 \%)$, renal pelvis invasion $(13 ; 7.4 \%)$, perirenal fat invasion $(30 ; 17.0 \%)$, and Gerota's fascia invasion $(4 ; 2.3 \%)$.

The clinicopathological features of patients are summarized in Table 1 based on the subtypes of RCC. It was agreed that chromophobe RCC should not be graded [17].

\section{Comparison of hTERT protein expression in RCC subtypes}

hTERT was expressed with variable intensities in the nucleus and partly in the cytoplasm of the RCC samples (Figure 1). Patients were classified into two groups as described earlier.

Of 176 RCC samples stained for hTERT, 4 (2.3\%) did not show any staining, whereas weak, moderate, and strong staining intensities were observed in 52 (29.5\%), 70 (39.8\%), and 50 (28.4\%) cases, respectively. The evaluation of hTERT expression based on the H- score values demonstrated that low expression of hTERT was found in $90(51.5 \%)$ and high expression was 
found in 86 (48.9\%) of RCC samples. Pearson's chi-square test showed a statistical significant association between hTERT expression and RCC subtypes (Pearson's $\chi 2, \mathrm{P}<0.001$ ) (Table 2).

The nonparametric Kruskal -Wallis \& Mann-Whitney $U$ tests were used to compare differences between hTERT expressions among the three tumor subtypes. Results of Kruskal -Wallis test indicated a statistically significant difference between the various levels of hTERT expression in different RCC subtypes ( $\mathrm{P}<0.001)$. Using the Mann-Whitney $U$ test, a statistically significant difference in the level of hTERT expression between ccRCC and chromophobe samples and also papillary and chromophobe RCC was found ( $<<0.001)$ (Figure 2).

\section{Associations between hTERT protein expression and clinicopathological characteristics in RCC}

Pearson's chi-square test showed that significant association between hTERT expression and tumor stage $(\mathrm{P}<0.001)$ as well as nuclear grade $(\mathrm{P}=0.028)$ in the ccRCC subtype. In addition, there was a significant association between higher levels of hTERT expression and tumor size (P $=0.050)$, MVI $(\mathrm{P}=0.005)$, lymph node invasion $(\mathrm{LNI})(\mathrm{P}=0.020)$, renal pelvis involvement $(\mathrm{P}=$ $0.010)$, renal sinus fat involvement $(\mathrm{P}=0.002)$, Gerota's fascia invasion $(\mathrm{P}=0.012)$, and distant metastasis $(\mathrm{P}=0.031)($ Table 3$)$.

Kruskal-Wallis test indicated that a statistically significant difference $(\mathrm{P}<0.001)$ between median hTERT expression and various tumor stages (I-IV). The median expression level of hTERT was 100 in stage I, 71 in stage II, 178 in stage III, and 238 in stage IV. Moreover, Mann-Whitney $U$ test showed highly significant difference in the levels of hTERT expression between stages I and IV $(\mathrm{P}<0.001)$ (Figure 3A).

The Kruskal-Wallis \& Mann-Whitney $U$ tests also showed statistically significant difference between hTERT expression and MVI $(\mathrm{P}=0.005)$ as well as LNI $(\mathrm{P}=0.030)$ (Figures 3B, 3C).

Bivariate analysis showed a significant direct correlation between hTERT expression and advanced tumor stage $(\mathrm{P}<0.001)$ as well as nuclear grade $(\mathrm{P}=0.049)$. In addition, statistically significant correlation was observed between hTERT expression and MVI $(\mathrm{P}=0.005)$, renal pelvis involvement $(\mathrm{P}=0.010)$, renal sinus fat involvement $(\mathrm{P}=0.002)$, Gerota's fascia invasion $(\mathrm{P}=$ 0.012), tumor size ( $\mathrm{P}=0.017)$, and distant metastasis $(\mathrm{P}=0.031)$.

In papillary RCC, there was significant association between hTERT expression and tumor stage $(\mathrm{P}=0.033)$, renal sinus fat involvement $(\mathrm{P}=0.009)$, and metastasis $(\mathrm{P}=0.010)$ (Table 4). In chromophobe RCC, we did not find any association between hTERT expression and clinicopathological features.

\section{Prognostic value of hTERT expression for clinical outcome in RCC}

Among 113 ccRCC samples included in this study, metastasis occurred in 28 (24.8\%) patients. During the follow-up period time, disease-related death was observed in 22 patients (19.5\%).

Kaplan-Meier survival analysis on these RCC patients, with median follow-up of 42 months (ranging from 1-77m) showed a significant difference between OS among the high and low hTERT expressing patient groups (Log Rank test, $\mathrm{P}=0.013$ ). The mean OS for patients with the high and low hTERT expression was $56(\mathrm{SD}=4.6)$ and $66(\mathrm{SD}=2.5)$ months, respectively (Figure 4A). 
The 5-year OS for the high and low hTERT expressing patients was $68 \%$ and $87 \%$, respectively $(\mathrm{P}=0.013)$.

The significant risk factors affecting the $\mathrm{OS}$ in univariate analysis included hTERT expression $(\mathrm{P}=$ $0.01)$, nuclear grade $(\mathrm{P}<0.002)$, tumor stage $(\mathrm{P}<0.001)$, and tumor size $(\mathrm{P}<0.001)$. Other clinicopathologic parameters did not significantly affect the OS in ccRCC patients. In multivariate analysis, tumor stage $(\mathrm{P}=0.044)$ and tumor size $(\mathrm{P}=0.011)$ were significantly related to the $\mathrm{OS}$ in ccRCC patients (Table 5).

In the papillary and chromophobe RCC patients, Kaplan-Meier survival analysis showed that high levels of hTERT expression is not significantly related to the OS(Figures 4B, C).

\section{Discussion}

Telomerase is an attractive and ideal target for therapy due to overexpression in the majority of malignancies and low or non-expression in most somatic cells [10,9].

Several studies have previously investigated the levels of telomerase activity and hTERT mRNA expression in RCC [12-14,18], whereas we could not find any study addressing the hTERT protein expression in RCC patients.

In the present study for the first time, the expression levels of hTERT protein was investigated in a well- characterized series of 176 RCC tissue samples from patients treated with radical nephrectomy. There was highly significant associations between the level of hTERT expression and RCC subtype. Moreover, a significant difference was observed in the levels of hTERT expression between ccRCC and chromophobe samples and also papillary and chromophobe RCC. These findings demonstrate that hTERT has different expression patterns among various subtypes of RCC, implying molecular mechanisms which lead to cellular immortality may differ according to tumor subtype [19].

In this study, we showed that hTERT protein expression is associated with more advanced tumor stage and higher tumor grade in ccRCC. The tumor stage and nuclear grade are the most reliable prognostic factors in RCC and tumors with a high Fuhrman grade have a more aggressive phenotype [20]. Importantly, we observed that the median expression of hTERT was higher in more advanced tumor stages (stage III and stage IV) when compared to the stages I and II, which depicts the association of hTERT protein expression with aggressiveness of ccRCC. Also, the tumor stage and Fuhram grade were significantly correlated with poor prognosis, however, tumor stage was found to be independent prognostic factor in multivariate analysis. The current study demonstrated a trend towards the association of increased hTERT protein expression and stage. Our result is in agreement with a study performed by Mekhail at al.[14] who showed that relationship between telomerase activity and tumor stage. In addition, significant association was found between the level of hTERT expression and distant metastasis. This observation is also consistent with a previous report based on the measurement of serum hTERT enzyme activity showing that its levels are associated with more advanced tumors stage and distant metastasis [21]. Moreover, the presence of statistically significant association between expression levels of hTERT and tumor size, renal pelvis and renal sinus fat involvement, and Gerota's fascia invasion showed that hTERT protein expression is related to the degree of malignancy and more advanced disease 
in ccRCC. Evidence shows that hTERT plays a role in tumor invasion and metastasis by promoting epithelial mesenchymal transition (EMT). Also, hTERT can activate the WNT/beta-catenin signaling pathway, therefore, it may contribute to cancer stem cell (CSC) maintenance [22]. CSCs are subpopulation among the tumor cells that have the ability to self-renew and have high tumorinitiating potential, and thus, they are able to drive cancer maintenance, progression, and metastasis [23]. Moreover, it is known that EMT generates cancer cells with stem cell-like characteristics; stem-like cells express markers associated with EMT; and the diversity and abundance of CSCs in solid tumors allows cells the ability to undergo EMT and cancer progression [24]. Castelo-Branco et al. showed that CSCs have significantly higher levels of hTERT expression and extremely short telomeres compared with the normal tissue stem cells [25]. CD133 as a CSC marker, was more highly expressed in hTERT-immortalized cells than in primary prostate cells [26].

In our study, tumor size was found to be independent prognostic indicator in multivariate analysis, in contrast with some previous studies which could not find any significant association between telomerase activity and tumor size $[13,27]$. Investigations have shown that tumor size has an important clinical and pathologic feature for patients with RCC and is significantly associated with risk of metastasis [28,29].

RCC is clinically recognized as a highly vascularized tumor, and one of the features of ccRCC is the presence of branching, thin-walled vessels in between the tumor cells [17]. Interestingly, we observed higher level of hTERT protein in this tumor subtype associated with higher MVI. Lang at al.[30] showed that MVI is related to cancer progression and survival in RCC and has more effects on prognosis compared to the macroscopic renal vein or vena cava invasion after treatment by radical nephrectomy. Several studies have reported the expression of hTERT as a potential factor in enhancing angiogenesis due to possible regulatory interactions with VEGF (Vascular endothelial growth factor)[31]. VEGF plays a pivotal role in tumor growth and progression in RCC $[32,33]$. LNI is also one of the other important prognostic factors in RCC. A previous study showed that LNI was independently associated with cancer specific survival after surgical resection and provides the strongest predictor of prognosis in patients without MVI [34].

Our results are in agreement with the previous studies which concluded that hTERT protein has an iportant role in advanced malignancy on some cancer types including breast cancer, gastric cancer, colorectal cancer, meningiomas, and spinal chordoma [35-39].

We found for the first time that tumors with higher expression of hTERT tend to have a worse prognosis compared to those with low expression. In addition, ccRCC patients who expressed higher level of hTERT had shorter 5-year overall survival compared with those with low expression. To our knowledge, this is the first study showing that hTERT protein expression is a prognostic marker in ccRCC, although without an independent significance in the multivariate analysis. Previous studies which focused on hTERT mRNA expression in RCC samples concluded that telomerase activity, was not related to prognosis [12-14]. A possible explanation for this discrepancy is post-transcriptional modification of hTERT mRNA.

In this study, we examined hTERT protein expression patterns in 32 papillary and 31 chromophobe RCC samples which are less frequent variants of RCC. We showed that in papillary RCC, there 
was significant association between increased hTERT protein expression and more advanced disease. However, there was not statistically significant association between hTERT protein expression and patients' outcomes. A larger sample size may be required to clearly address this issue. Moreover, in chromophobe RCC, no significant association was found between hTERT expression and tumor aggressiveness. This finding is in agreement with a previous study that telomerase was found only infrequently in chromophobe RCC [40]. ccRCC is known to be more aggressive and associated poorer prognosis than papillary and chromophobe RCC subtypes [41]. Almost all ccRCCs have deletions in the short arm of chromosome 3, resulting in mutations of the von Hippel-Lindau (VHL) gene which are frequent in ccRCC and are not observed in any other subtypes [42]. Evidence shows that mutations of VHL tumor suppressor gene leads to loss of function of the VHL tumor suppressor protein and consequent activation of the hypoxia pathway via the hypoxia-inducible factors [43] like transcription factor HIF1 $\alpha$ which plays an important role in development of ccRCC and upregulates a series of hypoxia-responsive genes including VEGF which has a pivotal role in ccRCC tumorigenesis [44]. Also, it has been previously reported that transcription factor HIF1 $\alpha$ induces hTERT expression and the level of hTERT is increased

under hypoxic conditions [45]. From these findings we are able to conclude that hTERT protein expression may be a novel prognostic indicator of worse outcome in patients with ccRCC, if follow up time is more prolonged. In addition, evaluation of hTERT protein expression indicate more aggressive tumor behavior and more advanced disease in ccRCC cases. Thus, evaluation of hTERT protein expression can be useful for predicting of tumor invasiveness in ccRCC patients.

\section{References}

1. Motzer RJ, Jonasch E, Agarwal N, Beard C, Bhayani S, Bolger GB, Chang SS, Choueiri TK, Costello BA, Derweesh IH (2015) Kidney cancer, version 3.2015. Journal of the National Comprehensive Cancer Network 13 (2):151-159

2. Eble J (2004) Classification of tumours: pathology and genetics of tumours of the urinary system and male genital organs. World Health Organization Classification of Tumours:255-257 3. Siegel R, Miller K, Jemal A (2017) Cancer Statistics, 2017. CA: a cancer journal for clinicians 67 (1):7-30

4. Ingels A, Zhao H, Thong AE, Saar M, Valta MP, Nolley R, Santos J, Peehl DM (2014) Preclinical trial of a new dual mTOR inhibitor, MLN0128, using renal cell carcinoma tumorgrafts. International journal of cancer 134 (10):2322-2329

5. Bitting RL, Healy P, Creel PA, Turnbull J, Morris K, Wood SY, Hurwitz HI, Starr MD, Nixon AB, Armstrong AJ (2014) A phase lb study of combined VEGFR and mTOR inhibition with vatalanib and everolimus in patients with advanced renal cell carcinoma. Clinical genitourinary cancer 12 (4):241-250

6. Lee-Ying R, Lester R, Heng DY (2014) Current management and future perspectives of metastatic renal cell carcinoma. International journal of urology 21 (9):847-855

7. Shigeishi H, Sugiyama M, Tahara H, Ono S, Kumar Bhawal U, Okura M, Kogo M, Shinohara M, Shindoh M, Shintani S (2011) Increased telomerase activity and hTERT expression in human salivary gland carcinomas. Oncology letters 2 (5):845-850

8. Pech MF, Garbuzov A, Hasegawa K, Sukhwani M, Zhang RJ, Benayoun BA, Brockman SA, Lin S, Brunet A, Orwig KE (2015) High telomerase is a hallmark of undifferentiated spermatogonia and 
is required for maintenance of male germline stem cells. Genes \& development 29 (23):24202434

9. Collins K, Mitchell JR (2002) Telomerase in the human organism. Oncogene 21 (4):564 10. Shay JW, Wright WE (2010) Telomeres and telomerase in normal and cancer stem cells. FEBS letters 584 (17):3819-3825

11. Cong Y, Shay JW (2008) Actions of human telomerase beyond telomeres. Cell research 18 (7):725

12. Yoshida Ki, Sakamoto Si, Sumi S, Higashi Y, Kitahara S (1998) Telomerase activity in renal cell carcinoma. Cancer 83 (4):760-766

13. Fujioka T, Hasegawa M, Suzuki Y, Suzuki T, Sugimura J, Tanji S, Koike H (2000) Telomerase activity in human renal cell carcinoma. International Journal of Urology 7 (1):16-21

14. Mekhail TM, Kawanishi-Tabata R, Tubbs R, Novick A, Elson P, Ganapathi R, Ganapathi M, Bukowski R Renal cell carcinoma (RCC) and telomerase activity: relationship to stage. In: Urologic Oncology: Seminars and Original Investigations, 2003. vol 6. Elsevier, pp 424-430 15. Trpkov K, Grignon DJ, Bonsib SM, Amin MB, Billis A, Lopez-Beltran A, Samaratunga H, Tamboli P, Delahunt B, Egevad L (2013) Handling and staging of renal cell carcinoma: the International Society of Urological Pathology Consensus (ISUP) conference recommendations. The American journal of surgical pathology 37 (10):1505-1517

16. Rasti A, Abolhasani M, Zanjani LS, Asgari M, Mehrazma M, Madjd Z (2017) Reduced expression of CXCR4, a novel renal cancer stem cell marker, is associated with high-grade renal cell carcinoma. Journal of cancer research and clinical oncology 143 (1):95-104

17. Delahunt B, Cheville JC, Martignoni G, Humphrey PA, Magi-Galluzzi C, McKenney J, Egevad L, Algaba F, Moch H, Grignon DJ (2013) The International Society of Urological Pathology (ISUP) grading system for renal cell carcinoma and other prognostic parameters. The American journal of surgical pathology 37 (10):1490-1504

18. Pal D, Sharma U, Singh SK, Kakkar N, Prasad R Inhibition of hTERT expression by MAP kinase inhibitor induces cell death in renal cell carcinoma. In: Urologic Oncology: Seminars and Original Investigations, 2017. Elsevier,

19. Bryan T, Englezou A, Gupta J, Bacchetti S, Reddel R (1995) Telomere elongation in immortal human cells without detectable telomerase activity. The EMBO journal 14 (17):4240

20. Ljungberg B, Cowan NC, Hanbury DC, Hora M, Kuczyk MA, Merseburger AS, Patard J-J, Mulders PF, Sinescu IC (2010) EAU guidelines on renal cell carcinoma: the 2010 update. European urology 58 (3):398-406

21. de Martino M, Taus C, Lucca I, Hofbauer SL, Haitel A, Shariat SF, Klatte T (2016) Association of human telomerase reverse transcriptase gene polymorphisms, serum levels, and telomere length with renal cell carcinoma risk and pathology. Molecular carcinogenesis 55 (10):14581466

22. Qin Y, Guo H, Tang B, Yang S-M (2014) The non-reverse transcriptase activity of the human telomerase reverse transcriptase promotes tumor progression. International journal of oncology 45 (2):525-531

23. Nguyen LV, Vanner R, Dirks P, Eaves CJ (2012) Cancer stem cells: an evolving concept. Nature reviews Cancer $12(2): 133$ 
24. Mani SA, Guo W, Liao M-J, Eaton EN, Ayyanan A, Zhou AY, Brooks M, Reinhard F, Zhang CC, Shipitsin M (2008) The epithelial-mesenchymal transition generates cells with properties of stem cells. Cell 133 (4):704-715

25. Castelo-Branco P, Zhang C, Lipman T, Fujitani M, Hansford L, Clarke I, Harley CB, Tressler R, Malkin D, Walker E (2011) Neural tumor-initiating cells have distinct telomere maintenance and can be safely targeted for telomerase inhibition. Clinical Cancer Research 17 (1):111-121

26. Miki J, Furusato B, Li H, Gu Y, Takahashi H, Egawa S, Sesterhenn IA, McLeod DG, Srivastava S, Rhim JS (2007) Identification of putative stem cell markers, CD133 and CXCR4, in hTERTimmortalized primary nonmalignant and malignant tumor-derived human prostate epithelial cell lines and in prostate cancer specimens. Cancer research 67 (7):3153-3161

27. Sugimura K, Yoshida N, Hisatomi H, Nakatani T, Ikemoto S (1999) Telomerase activity in human renal cell carcinoma. BJU international 83:693-697

28. Thompson RH, Hill JR, Babayev Y, Cronin A, Kaag M, Kundu S, Bernstein M, Coleman J, Dalbagni G, Touijer K (2009) Metastatic renal cell carcinoma risk according to tumor size. The Journal of urology $182(1): 41-45$

29. Herrlinger A, Schott G, Schafhauser W, Schrott K (1992) The significance of tumor diameter in renal cell carcinoma. Der Urologe Ausg A 31 (2):70-75

30. Lang H, Lindner V, Letourneux H, Martin M, Saussine C, Jacqmin D (2004) Prognostic value of microscopic venous invasion in renal cell carcinoma: long-term follow-up. European urology 46 (3):331-335

31. Hartwig FP, Nedel F, Collares TV, Tarquinio SB, Nör JE, Demarco FF (2012) Telomeres and tissue engineering: the potential roles of TERT in VEGF-mediated angiogenesis. Stem Cell Reviews and Reports 8 (4):1275-1281

32. Jacobsen J, Grankvist K, Rasmuson T, Bergh A, Landberg G, Ljungberg B (2004) Expression of vascular endothelial growth factor protein in human renal cell carcinoma. BJU international 93 (3):297-302

33. Albiges L, Salem M, Rini B, Escudier B (2011) Vascular endothelial growth factor-targeted therapies in advanced renal cell carcinoma. Hematology/oncology clinics of North America 25 (4):813-833

34. Zubac DP, Bostad L, Seidal T, Wentzel-Larsen T, Haukaas SA (2008) The prognostic relevance of interactions between venous invasion, lymph node involvement and distant metastases in renal cell carcinoma after radical nephrectomy. BMC urology 8 (1):19

35. Poremba C, Heine B, Diallo R, Heinecke A, Wai D, Schaefer KL, Braun Y, Schuck A, Lanvers C, Bankfalvi A (2002) Telomerase as a prognostic marker in breast cancer: high-throughput tissue microarray analysis of hTERT and hTR. The Journal of pathology 198 (2):181-189

36. Gigek CO, Leal MF, Silva PNO, Lisboa LCF, Lima EM, Calcagno DQ, Assumpcao PP, Burbano RR, Smith MdAC (2009) hTERT methylation and expression in gastric cancer. Biomarkers 14 (8):630-636

37. Li J, Cao X, Fang Y, Liao Z-E, Liu Y-Y, Huang B-D, Han Y-J (2013) Overexpression of hTERT in potentially malignant colorectal laterally spreading tumors. Molecular medicine reports 7

(5):1409-1412

38. Maes L, Kalala J, Cornelissen R, De Ridder L (2006) Telomerase activity and hTERT protein expression in meningiomas: an analysis in vivo versus in vitro. Anticancer research 26 (3B):2295-2300 
39. Zou M-X, Lv G-H, Li J, She X-L, Jiang Y (2016) Upregulated human telomerase reverse transcriptase (hTERT) expression is associated with spinal chordoma growth, invasion and poor prognosis. American journal of translational research 8 (2):516

40. Kinoshita H, Ogawa O, Mitsumori K, Kakehi Y, Terachi T, Yoshida O (1998) Low frequency of positive telomerase activity in a chromophobe subtype of renal cell carcinoma. The Journal of urology 159 (1):245-251

41. Lau WK, Cheville JC, Blute ML, Weaver AL, Zincke H (2002) Prognostic features of pathologic stage T1 renal cell carcinoma after radical nephrectomy. Urology 59 (4):532-537

42. Hansel D (2006) Genetic alterations and histopathologic findings in familial renal cell carcinoma. Histology and histopathology 21 (4):437-444

43. Clark PE (2009) The role of VHL in clear-cell renal cell carcinoma and its relation to targeted therapy. Kidney international 76 (9):939-945

44. Klatte T, Seligson DB, Riggs SB, Leppert JT, Berkman MK, Kleid MD, Yu H, Kabbinavar FF, Pantuck AJ, Belldegrun AS (2007) Hypoxia-inducible factor $1 \alpha$ in clear cell renal cell carcinoma. Clinical Cancer Research 13 (24):7388-7393

45. Nishi H, Nakada T, Kyo S, Inoue M, Shay JW, Isaka K (2004) Hypoxia-inducible factor 1 mediates upregulation of telomerase (hTERT). Molecular and cellular biology 24 (13):6076-6083

\section{Legends for figures}

Figure 1. Immunohistochemical analysis of hTERT expression in renal cell carcinoma (RCC) samples. hTERT expression in clear cell RCC: high expression (A, A-1) and low expression (B, B-1). hTERT expression in papillary RCC: $(C, C 1)$ and in chromophobe RCC (D, D1). Figures are shown with a magnification of $\times 100$ and $\times 200$

Figure 2. Analysis of hTERT expression level in different renal cell carcinoma (RCC) subtypes including clear cell, chromophobe, and papillary RCC using Mann-Whitney $U$ test. Based on the standard definitions, each box-plot shows the median (bold line), interquartile line (box), and outlier observation (circle).

Figure 3. Box plot analysis of hTERT expression levels in stage I to IV (A), microvascular invasion (MVI) (B), and lymph node invasion (LNI) (C) in ccRCC, using Mann-Whitney $U$ test. Based on the standard definitions, each box-plot shows the median (bold line) and interquartile lines (box).

Figure 4. Kaplan-Meier curves for overall survival (OS) based on hTERT protein expression level in ccRCC. In ccRCC patients, higher level of hTERT protein expression was associated with shorter overall survival compared to the tumors with low expression of this protein (A). In the papillary and chromophobe RCC patients, Kaplan-Meier survival analysis showed that high levels of hTERT expression is not significantly related to the OS (B, C). 